stage. While not removing the problems associated with non-ideality, it will tend to reduce differences between calibration and prediction. To do this of course is not always possible, particularly where the effect of changing dyebath conditions is under consideration, e.g. liquor ratio, electrolyte concentration, $\mathrm{pH}$ and dye concentration. To attempt calibration experiments to adequately cover the results of such changes would remove the need for prediction. If one of the changes in conditions anticipated is that of temperature then obviously the calibration experiments would have to include a measure of the heats of dyeing of the dyes concerned, so that the affinities used would be correct or correctable to the temperatures involved.

It may be considered that the treatment developed presents something of an 'over-kill', particularly in terms of the determination of the amounts of all the different ionic species in the system originating from a variety of sources. For many dyeing systems this is indeed true, i.e. in dyebaths of high liquor ratio and in the presence of moderate to high concentrations of added electrolyte, where the adventitious contribution of ions from other sources could be negligible. On the other hand there are other cases where such considerations would be warranted, e.g. when dyeing a fibre such as wool, which has a considerable potentiality to contribute ions, at low liquor ratios with the minimum addition of electrolyte. Since the application of the treatment clearly calls for the use of a computer there should be no problems in covering all eventualities, apart from the increased complexity of the computer program. However, once written, the program can be used in the general sense if so desired or in a suitably modified way by using the appropriate data input.

The equations produced are complex and if the only method of evaluation available was by manual calculation then the time required would be a serious obstacle to their usefulness in practical circumstances. However, the continuing development of computers has totally removed this difficulty, so much so that the practical use of these methods could well be an online procedure once the necessary computer programs are available.

\section{REFERENCES}

1. H H Sumner, J.S.D.C., 102 (1986) 301.

2. H H Sumner, J.S.D.C., 102 (1986) 341.

\title{
Foam Generation in Rotor-Stator Mixers
}

\section{A B J Kroezen and J Groot Wassink}

Twente University of Technology

Enschede

The Netherlands

One of the unit operations in foam technology is foam generation in a liquid/gas mixer. The foaming process of an aqueous liquid system with a surface-active agent in a rotor-stator mixer has been studied, and it was found that it could be represented by a mixing characteristic relating the liquid flow rate to the maximum gas flow rate needed to produce a foam without 'blow-by'. Too high a gas flow rate resulted in a short-circuiting flow of gas via the rotor shat to the outlet. The influence of the number of rotor-stator blades, equipment size and operating pressure on the behaviour of the foam mixer has been investigated.

\section{INTRODUCTION}

There is an increasing interest in using foam as a medium for distributing small quantities of chemicals over porous substrates such as textiles, carpets and nonwovens. The substitution of foams for water in dyeing, printing and finishing can result in considerable savings in the amount of water used and in the energy required to evaporate water from treated fabrics. In many cases wet fabrics may be dyed or finished without an intermediate drying operation. Also since less water is used, there is less migration during drying, giving superior results $[1,2]$.

Three steps can be distinguished in foam application equipment: firstly the generation of foam in the mixer, secondly transport and distribution of foam in the piping system and finally application of the foam to the substrate.

\section{Foam Generation}

In a foam mixer kinetic energy is supplied to mix a gas phase and a liquid phase under foaming conditions. The liquid phase is usually water containing surface-active agents, thickeners and chemicals to be applied to the substrate. In most cases air is the gas phase. The structure of foam may be characterised by the blow ratio and the bubble size distribution. The blow ratio is the volumetric ratio of gas and liquid in the foam. The bubble size distribution is a measure of the degree of dispersion of the foam. The former can be controlled by the gas and liquid flow rates supplied to the mixer, while bubble size is a function of the energy supply to the mixer; a high energy supply per unit volume gives rise to small bubbles in the mixer [3].

There are two types of foam mixers: the rotor-stator mixers and the static mixers. The energy supply to rotorstator mixers is determined by the energy transferred from the revolving blades to the foam mixture. In static mixers the energy supply is related to the flow rate of the foam. The energy consumption in static mixers depends to a great extent on the production rate of foam (which is proportional to the foam flow rate in the mixer), whereas rotor-stator mixers are capable of producing foam with a consistent structure over a wide range of foam production rates.

\section{Transport and Distribution of Foam}

The piping system in foam application equipment has three functions: transport of the foam from the mixer to the substrate, distribution of the foam over the width of the substrate and build-up of a given pressure in the mixer. Destabilisation of foam in transport and distribution units can be greatly influenced by the design and layout of the equipment, as well as the flow rates in the unit. As a result of drainage, long residence times lead to a high degree of non-uniformity of the foam. High shear rates induced by high flow rates or by sharp edges in the distribution unit 
may lead to destruction of the foam. It is frequently advantageous to define a maximum shear rate above which foams are destroyed.

In several systems, especially in closed ones, distribution of the foam over the width of the substrate is affected by the flow resistance of the distribution unit. The geometry of the distribution unit in combination with the rheological properties of the foam (foam has a high viscosity, in comparison with the liquid) determines the width distribution of foam on the substrate.

Compressibility is another important property of foam. The high pressure drop between the entry to and the exit from the transport and distribution unit calls for a high pressure in the mixer, which in turn gives rise to a lower blow ratio in the mixer. The foamability (defined as the maximum blow ratio that can be produced) is a function of the mixer pressure. A high pressure in the mixer causes a high expansion factor of the foam in the transport and distribution unit.

\section{Application to the Substrate}

In foam application a relatively small amount of liquid is distributed over the substrate. In the generation of the foam the small amount of liquid is volumetrically enlarged by a factor of between five and 20 . In most cases the foam will be quickly destroyed when it comes into contact with a hydrophilic substrate. The liquid is absorbed by the substrate and the gas escapes. The destruction of the foam may be intensified by mechanical and thermal forces.

\section{FOAMABILITY AND FOAM STABILITY}

Gas emulsions are colloids, with the gas being the dispersed phase and the liquid the continuous phase. Gas emulsions with a high volume fraction of gas (50-90\%) are called foams $|4|$. As already mentioned the structure of foams can be characterised by the blow ratio (the volume ratio between gas and (iquid) and the bubble size distribution. Foams are thermodynamically unstable. Structure deformation as well as phase separation are caused by their high surface energy in combination with the effects of gravity. Structure deformation is the result of drainage of the liquid from the lamellae as well as gas diffusion from small bubbles to large bubbles and the consequent bursting of the lamellae.

The destabilisation rate is determined by the bulk and surface properties of the liquid, the foam structure and also by mechanical and thermal stresses in the foam $[4,5]$.

Foamability is a term used to quantify the net outcome between surface creation and surface destruction. For a given liquid system the foamability is experimentally measured as the foam volume obtained under standard foaming conditions.

Bikerman [2] reviewed several methods to quantity foamability. Two commonly used methods are the pour test and the column test. In the pour test (Ross-Miles method standardised by the American Society of Testing Materials [6l) a given volume of liquid is poured from a certain height onto a column of liquid. The height of foam formed is a measure for the foamability. In the column test a constant gas flow is supplied at the bottom of a column of liquid, the foam height obtained again giving the foamability $[7]$. This property is measured in order to quantity the foaming properties of the liquid system and is dependent on the test method used; hence it has only a relative value |5|. Therefore foamability can better be taken as a process parameter, determined on the one hand by the foaming properties of the liquid system and on the other by the mixing conditions in the equipment used.

Foamability of liquid systems is an indication of the foaming rate as well as of the maximum attainable blow ratio.
Mixing characteristics as reported in this paper are very important as a measure for foamability. The differences in process parameters in foam mixers and in standardised tests may be so substantial that the latter cannot be used to describe the foamability in liquid/gas mixers. The foamability should therefore be measured under the same mixing conditions as in commercially applied foam mixers. The principle of similarity in mixing characteristics is important when scaling-up foamability measurements based on laboratory trials.

\section{DESCRIPTION OF EQUIPMENT}

For the experiments standard foaming agents, Diphasol EA and Diphasol VA (CGY) were used. The composition (by weight) of the liquid system was $1.5 \%$ Diphasol EA, $1 \%$ Diphasol VA, $0.1 \%$ acetic acid and $97.4 \%$ water. Air was used as a gas phase.

The mixer is of the rotor-stator type. It has an annular space provided with a number $(n)$ of rotor-stator units. A rotor-stator unit is the mixing space enclosed by one rotor blade and one stator blade, and every rotor or stator blade has a certain number of mixing pens. The elementary unit of the mixer is shown in Figure 1. The geometry of the mixer is determined by the number of rotor-stator units, and the diameter and the dimensions of the elementary unit.

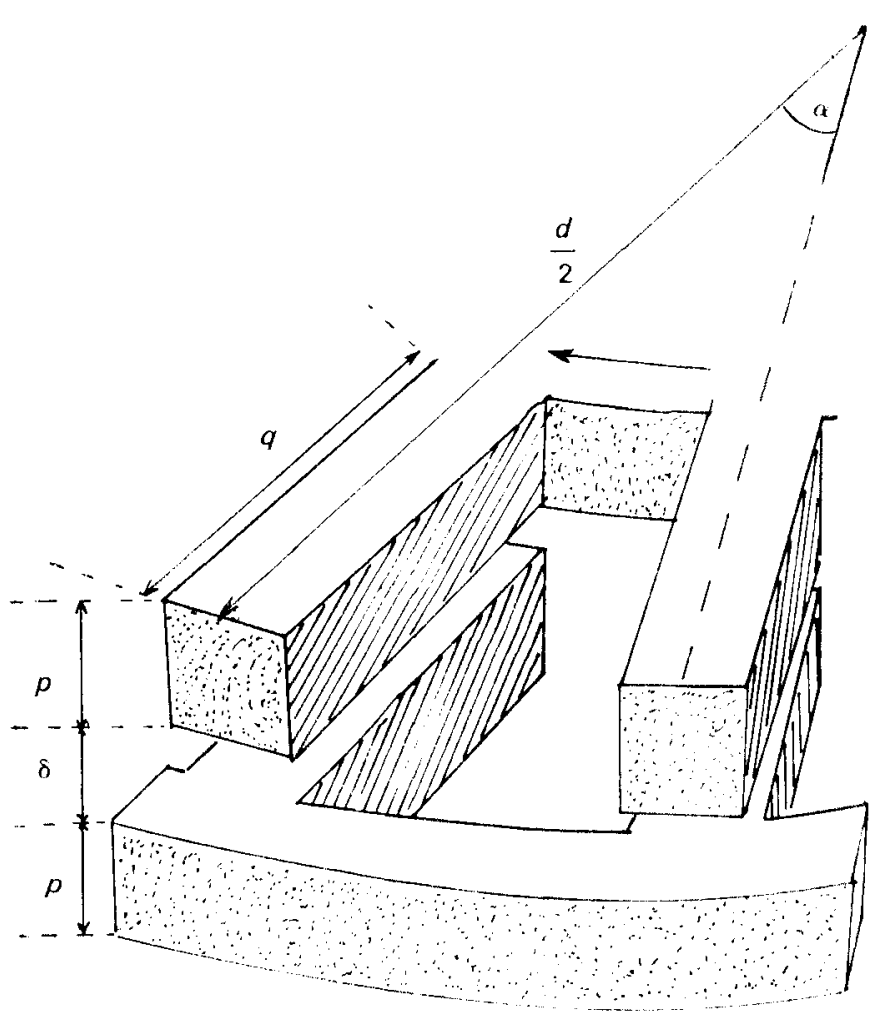

Figure 1-Elementary unit of the mixer

In order to examine the scaling-up procedures for foam mixers of a given geometry of elementary unit, the number of rotor-stator units and the diameter of the mixer can be varied. The number of rotor-stator units can be changed easily by replacing a certain number of rotor-stator blades in the 'fill-up' body. Two mixers were used having different radii. Mixer 1 has a diameter of $62 \mathrm{~mm}$ with 18 pens and mixer 2 has a diameter of $130 \mathrm{~mm}$ with 40 pens on each rotor or stator blade. The various properties of the mixing equipment are given in Table 1. In Figure 2 the design of mixer 2 is shown. Mixer 1 has a similar construction but with a smaller radius. 


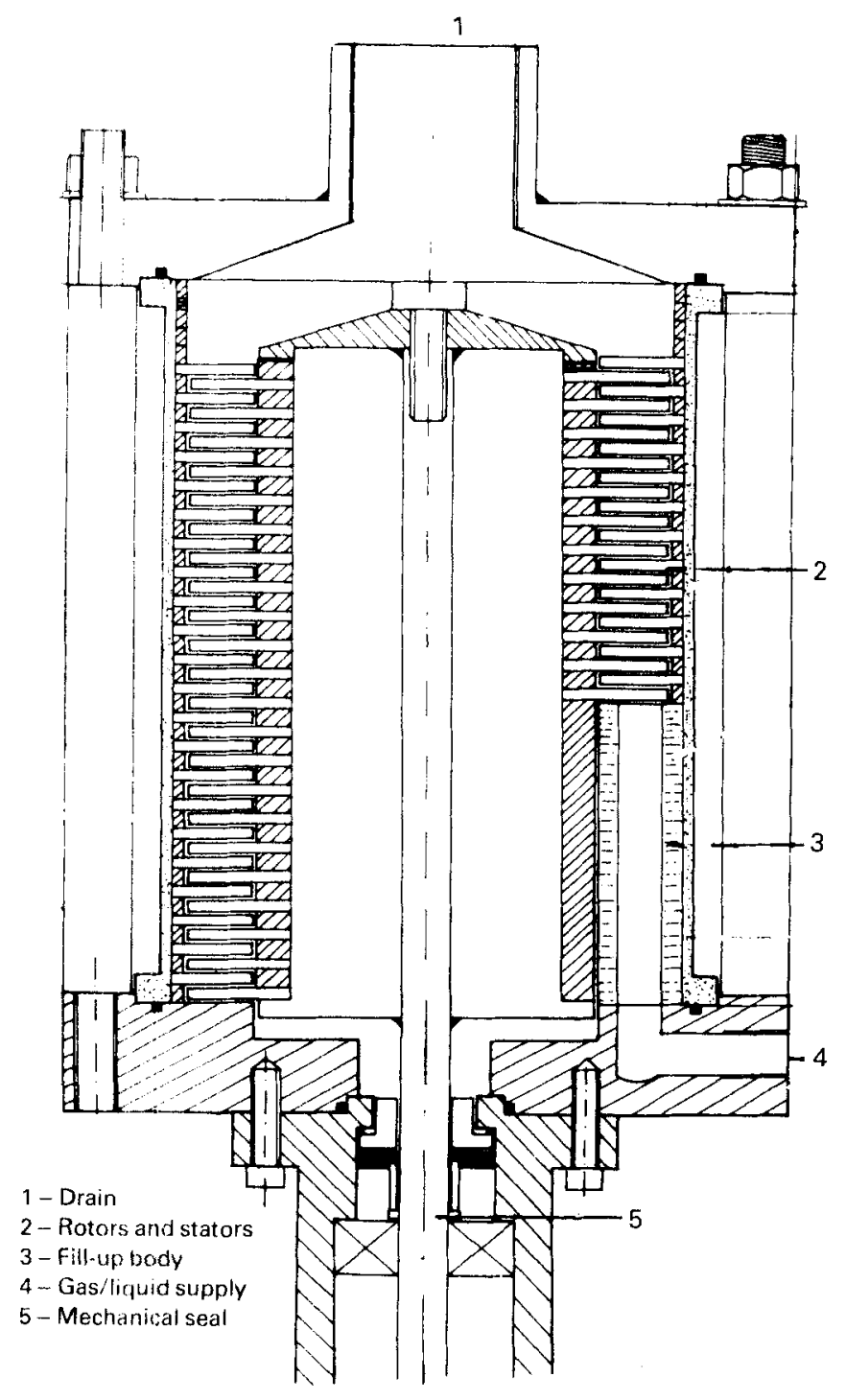

TABLE 1

Mixer Parameters

\begin{tabular}{|c|c|c|c|c|c|c|c|}
\hline & $\underset{(\mathrm{mm})}{p}$ & $\begin{array}{c}q \\
(\mathrm{~mm})\end{array}$ & $\begin{array}{c}\delta \\
(\mathrm{mm})\end{array}$ & $\begin{array}{c}d \\
(\mathrm{~mm})\end{array}$ & $\alpha$ & $\begin{array}{c}v \\
\langle\mathrm{~m} / \mathrm{s}\rangle\end{array}$ & $n$ \\
\hline Mixer 1 & 3 & 17 & 1 & 62 & $360^{\circ} / 18$ & $1-6$ & $3-10$ \\
\hline Mixer 2 & 3 & 17 & 1 & 130 & $360^{\circ} / 40$ & $3.3-10$ & $4-23$ \\
\hline
\end{tabular}

Gas and liquid can be supplied separately to one side of the mixer, with the foam generated flowing in the transport pipe on the opposite side. The outlet, being relatively large and without sharp edges, prevents destruction of the foam.

Figure 3 shows the equipment used for the measurements. A monopump is used to transport the liquid from the store vessel to the mixer. The flow rate is measured by flowmeters. The gas flow is adjusted by a flow controller and measured under a constant gauge pressure of $5 \mathrm{~atm}$.

The pressure in the mixer attributable to flow resistance in the transport pipe was measured. The number of revolutions of the mixer is controlied by a frequency transformer.

Mix characteristics were measured by determining the maximum rate of flow of gas which can be dispersed in the liquid phase at a certain liquid flow rate. The transition from producing a homogeneous foam to one with 'blow-by' is very sharp. The blow-by can be discerned visually as large bubbles in the foam with diameters of approximately $0.5-5$ $\mathrm{cm}$.

\section{GENERAL PROCESS ANALYSIS}

The foamability of a given liquid system in a foam mixer is limited by a maximum blow ratio and the foaming capacity. At a given liquid flow rate gas can only be dispersed into a foam at a limited flow rate. Too high a gas flow rate results in blow-by of unmixed air in the outlet.

Figure 2 -Mixer 2
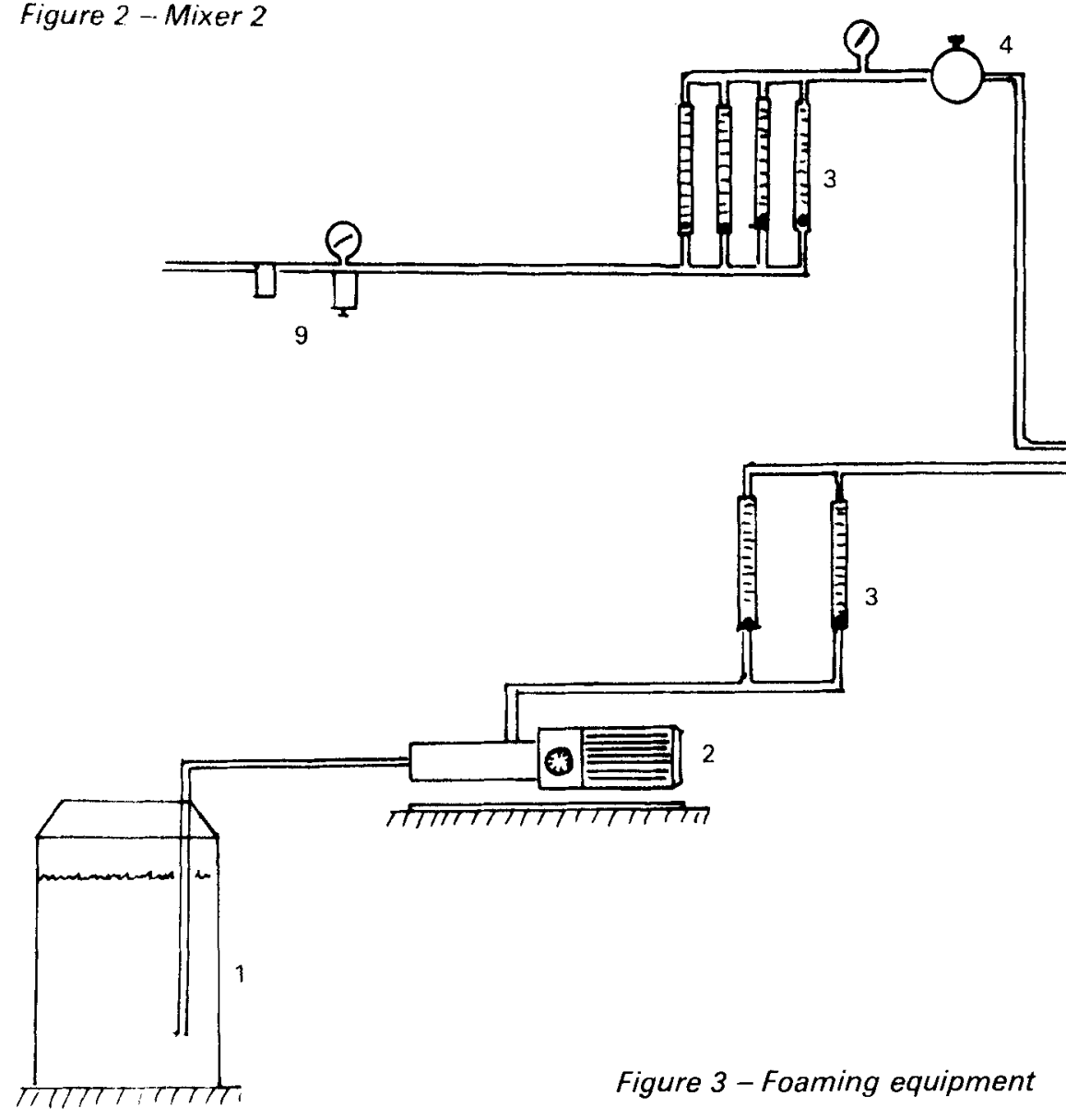
Normally foam is transported from the mixer by tube, a mixer pressure being built up by the pressure drop over the length of the transport tube. Gas and liquid are mixed under pressure and subsequently expanded in the transport tube, so that blow ratios much higher than those produced in the mixer can be obtained. The expansion of the foam must be carried out very smoothly. High shear rates in the expansion section, caused by high flow rates and sharp edges in the transport tube, will lead to destruction of the foam. The apparent toamability is different for mixers with tubes having different pressure drops.

A determination of the foamability independent of mixer pressure can be obtained if the compressibility of the foam is taken into account. This can be calculated approximately using the ideal gas law for the gas phase and assuming incompressibility for the liquid phase. The blow ratio, $B_{0}$, at the mixer end of the tube is given by Eqn 1:

$$
B_{\mathrm{o}}=\phi_{\mathrm{go}} / \phi_{1}
$$

The actual blow ratio, $B_{m}$ in the mixer, taking into account the compressibility of the foam is given by Eqn 2:

$$
B_{\mathrm{m}}=\phi_{\mathrm{gm}} / \phi_{1}
$$

The blow ratio in the mixer can be calculated from the gas flow rate and the corresponding values at the mixer end of the tube (Eqns 3 and 4 ):

$$
\begin{gathered}
\phi_{\mathrm{gm}}=\left(P_{\mathrm{o}} / P_{\mathrm{m}}\right) \phi_{\mathrm{go}} \\
B_{\mathrm{m}}=\left(P_{\mathrm{o}} / P_{\mathrm{m}}\right) B_{\mathrm{o}}
\end{gathered}
$$

The maximum rate of flow at which gas can be dispersed in the liquid (as a function of the liquid flow rate) is represented by a so-called mixing characteristic. The mixing characteristic delimits the working area of the mixer. Higher gas flow rates result in blow-by. A typical example of a mixing characteristic is given in Figure 4.

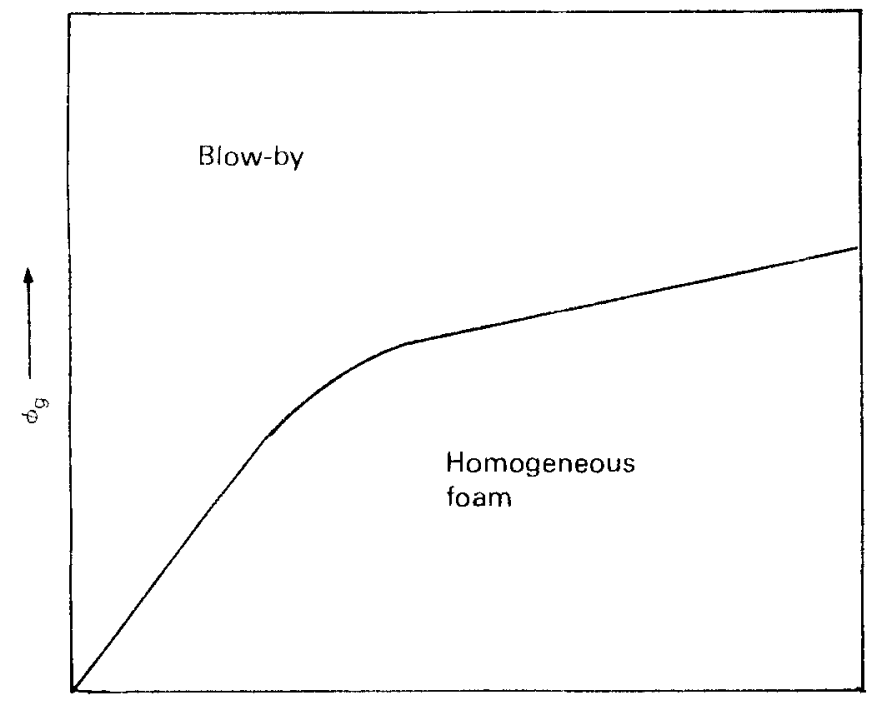

$$
\phi_{1} \longrightarrow
$$

\section{Figure 4-Mixing characteristic}

The mixing characteristic may be approximated by two straight lines, the so-called equilibrium and capacity lines. The capacity line represents the capacity limitation of the mixer and the equilibrium line represents the maximum blow ratio that can be obtained in the mixer. The form of the mixing characteristic as given in Figure 4 may be explained in terms of two phases being distinguishable in the mixer, a continuous gas phase and a foam phase. As a result of the centrifugal forces a gaseous envelope is formed around the rotor shaft, distinct from the foam phase (Figure 5). The size of this air envelope is determined by the gas flow rate as well as by the rate of transfer of gas to the foam phase in the mixer.

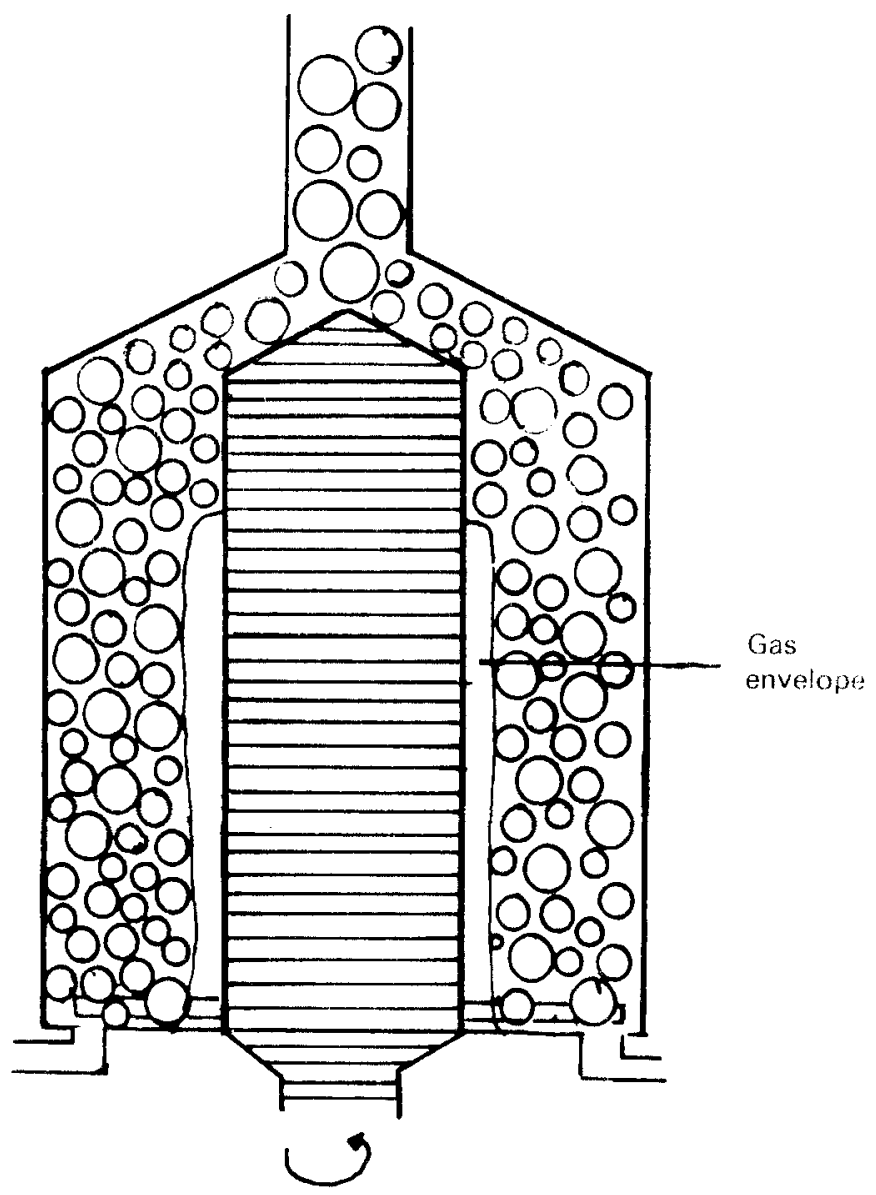

Figure 5 - Gas envelope in the mixer

If the flow of gas is limited, only a few rotor-stator units are actually used for mixing. When the gas flow rate is higher than can be accommodated by the total mixing capacity of the mixer, unmixed gas will leave the mixer. At relatively long residence times in the mixer the maximum blow ratio that can be produced is determined by an equilibrium between mixing and separation of foam. The net rate of transfer of gas from the gas envelope to the foam phase is then zero. In Figure 6 mixing characteristics for different numbers of rotor-stator units are represented. All the mixing characteristics have the same equilibrium line but different capacity lines. The shape of the mixing characteristic is controlled by three factors. The first of these represented by the equilibrium line indicating the $\max$ imum blow ratio which can be produced in the mixer, $B_{\theta}$ determined by an equilibrium between mixing and unmixing. Two other factors are responsible for the position of the capacity line. Firstly mixing capacity of each rotor-stator unit is quite constant and may be characterised by the gas flow rate that can be dispersed per rotor-stator unit. The remaining factor is the mixing of gas and liquid just after entering the mixer. The gas flow is distributed over the foam phase and the gas envelope around the rotor shaft. The gas initially dispersed in the foam phase may be described by an initial mixing blow ratio, $B_{0}$ 


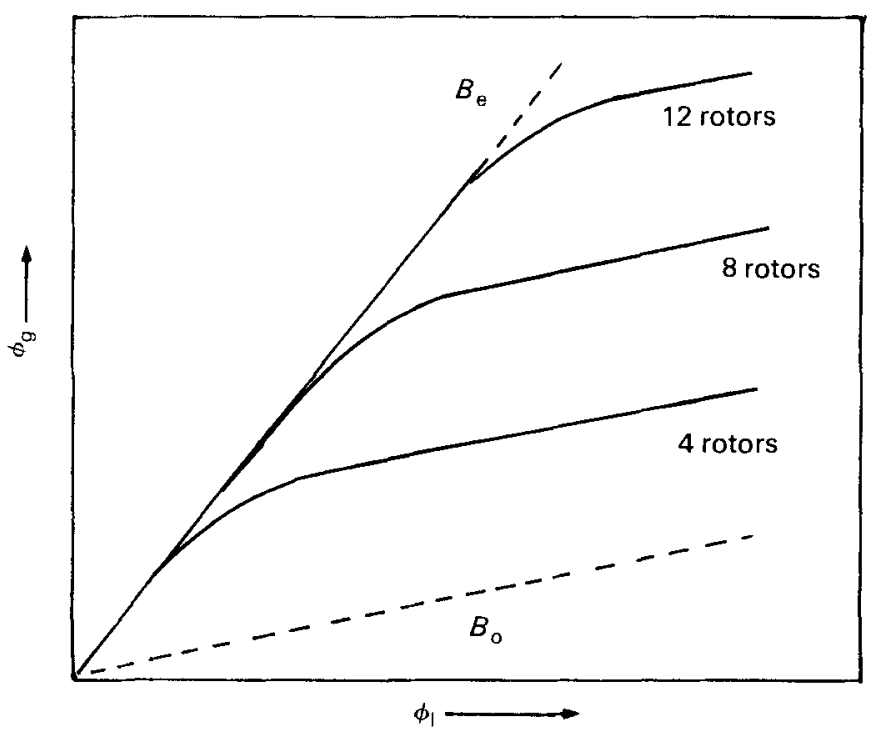

Figure 6 - Mixing characteristics for different number of rotor-stator units

The number of rotor-stator units required for a given operation can be determined by the total mixing capacity of the rotors $(n \phi)$. The values of $B_{0}$ and $B_{e}$ are not affected by the number of rotor-stator units. The mixing capacity of a mixer with a series arrangement of $n$ rotor-stator units has the same capacity as a parallel arrangement of two mixers with a series arrangement of $n / 2$ rotor-stator units.

\section{EXPERIMENTAL RESULTS}

Mixing characteristics of the foaming process of a liquid system with Diphasol EA and Diphasol VA in the mixer already described were measured as a function of diameter, number of rotor-stator units, number of revolutions and mixer pressure.

The mixing characteristics were found to be independent of pressure if the compressibility was determined using pressure drops over the length of tube of up to $4 \mathrm{~atm}$. All measurements under constant mixer conditions with different pressure drops over the tube gave rise to the same mixing characteristics. In Figure 7 several measured mixing characteristics are represented.

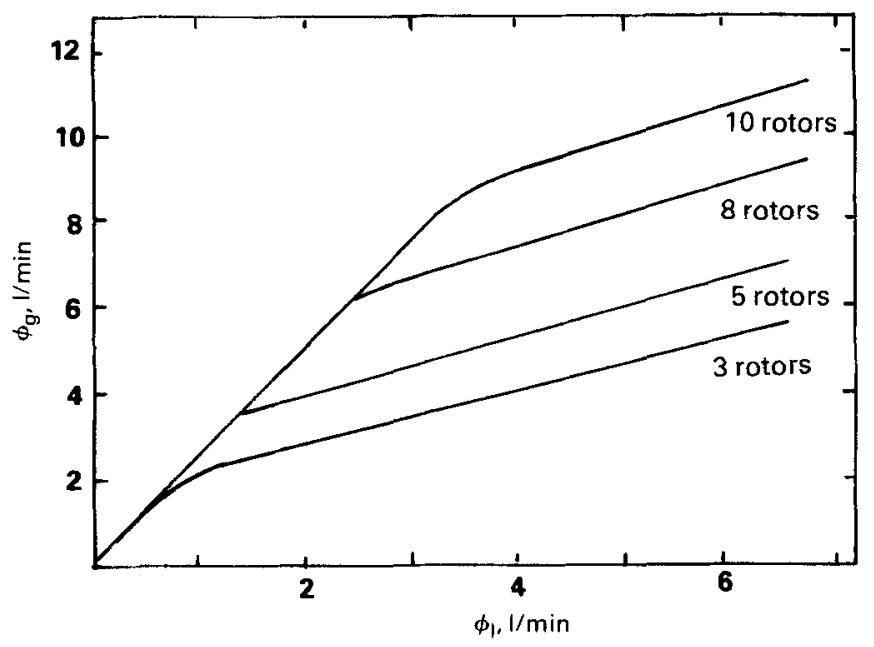

Figure 7 - Mixing characteristic of mixer 1, rotor tip velocity $=6 \mathrm{~m} / \mathrm{s}$

In order to calculate the compressibility of the gas phase in the foam using the ideal gas law, it was assumed that the solubility of gas in the liquid is pressure dependent and may be described by a Henry coefficient $(H=P / C)$. This effect will increase the expansion in the transport tube. Using air as the gas phase and aqueous liquid phases, the change in solubility as a function of pressure between 1 to 5 atm does not appreciably affect the compressibility of foam.

A second assumption made was to neglect the pressure increase in the bubbles due to the surface tension. If $y$ is the surface tension and $r$ is the radius of the bubble, the pressure excess will be $\Delta P(=2 \gamma / r)$. The liquid system used had a surface tension of $0.03 \mathrm{~N} / \mathrm{m}$ and the mean bubble diameter of the foam produced ranged from 30 to $150 \mu \mathrm{m}$ so that this effect indeed may be neglected. A third important aspect is the destruction of the foam which may occur at high shear rates in the transport tube. The foam is separated and gas bubbles are formed with diameters much higher than the mean diameter of the foam bubbles.

Destruction of foam in the transport tubes decreases the apparent foamability and the measured mixing characteristics are no longer independent of pressure.

Several mixing characteristics are represented in Figure 7. The equilibrium line and the capacity line are represented by two quite distinct straight lines; the transition from the equilibrium line to the capacity line is remarkably sharp. It is not likely that the transition from the capacity line to the equilibrium line is due only to the increasing rate of separation of mixed gas in the foam into the gas envelope. It seems to be that at the equilibrium blow ratio the rate of mixing is also reduced by a change in hydrodynamic behaviour. As already mentioned, a mixing characteristic may be represented by three parameters: the equilibrium blow ratio $\left(B_{\mathrm{e}}\right)$, the mixing capacity per rotor $(\phi)$ and the initial mixing blow ratio $\left(B_{0}\right)$. These parameters are independent of the rates of gas and liquid input. This behaviour was observed if the ratio of the net upward foam velocity in the mixer and the rotor velocity was much less than unity. In Figure 8 the measured values of $B_{0}, B_{\mathrm{e}}$ and $\phi$ are represented as a function of the tip velocities of the rotor blades for two mixers with different diameters. The comparison between the two mixers is based on corresponding tip velocities of the rotor blades and not on the corresponding number of revolutions, because the energy dissipation per unit volume is equal at equal tip velocities. At tip velocities lower than $3 \mathrm{~m} / \mathrm{s}$ the foam had a mean bubble size larger than $200 \mu \mathrm{m}$ and was very unstable. In this region only $B_{\mathrm{e}}$ could be accurately determined. At the overlapping tip velocities of mixer 1 and mixer 2 the initial blow ratios and also the equilibrium blow ratios had about the same value. The mixing capacity per rotor-stator unit of mixer 2

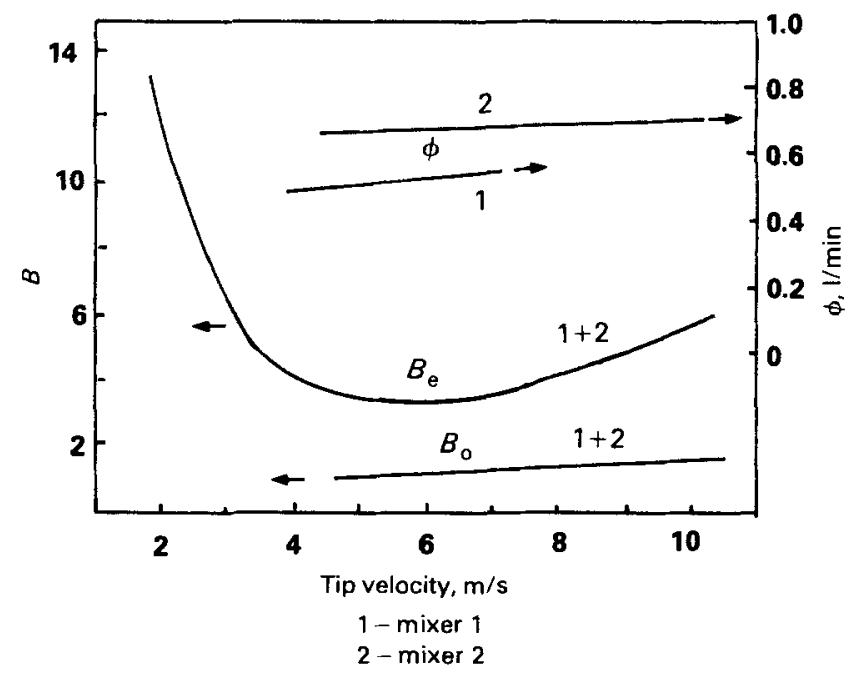

Figure 8 - mixing parameters of mixers 1 and 2 
was $20 \%$ higher than that of mixer 1 . In view of the number of mixing pens per rotor blade or the mixing per volume per rotor-stator unit, mixer 1 had a larger mixing capacity in comparison with mixer 2 . Therefore scaling-up of the mixer diameter would be an ineffective method of increasing the capacity of the mixer. An explanation of the lower mixing capacity per unit volume of mixer 2 could be the lower centrifugal forces in this mixer at the same tip velocities.

The initial mixing blow ratio and the rate of mixing per rotor-stator unit both increased slowly at increasing tip velocities. The equilibrium blow ratio had a minimum value at a tip velocity of 4-5 m/s. In the work reported only one liquid system with a surface-active agent was tested. However, the results seem to have a more general character. Although only verified for one foaming system, the results as represented in Figure 8 are typical process parameters for the aqueous Diphasol EA/VA solution in the mixer used.

\section{CONCLUSIONS}

1. Foamability may be satisfactorily represented by a mixing characteristic, which depends on the mixing conditions and the properties of the liquid system.

2. The increase in mixer capacity by processing under pressure and expanding the foam in the transport tube can be calculated by taking into account the compressibility of the gas phase. The mixer pressure does not affect the mixing characteristic.

3. From the experiments it may be concluded that the capacity increases linearly with the number of rotorstator units.

4. Scaling-up the dimensions of a foam mixer does not lead to similar mixing characteristics.
5. The experiments were carried out with one liquid system in one type of mixer. It may be expected that the mixing characteristics are dependent on mixing conditions and properties of the liquid.

\section{REFERENCES}

1. T F Cooke, Text. Chem. Colorist, 15 (5) (1983) 74

2. T L. Dawson, J.S.D.C., 97 (1981) 262.

3. P H Calderbank, Chem. Eng., (1967) 209

4. A J De Vries, PhD thesis (University of Delft, 1957).

5. J J Bikerman, 'Foams' (Heidelberg: Springer Verlag, 1973).

6. ASTM Test Method D1173-53 (1970).

7. ASTM Test Methods D892-63 (1968) and D1881-67.

\section{List of Symbols}

$B=$ blow ratio

$\phi=$ flow rate

$P=$ pressure

$p, q, \delta=$ dimensions of elementary unit of mixer

$d=$ diameter of mixer

$\alpha=$ angle between two rotor pens

$v=$ tip velocity of rotor blades

$n=$ number of rotor-stator units

$r=$ radius of bubbles

$\gamma=$ surface tension

\author{
Subscripts \\ $\mathrm{e}=$ equilibrium \\ $\mathrm{g}=\mathrm{gas}$ \\ I=liquid \\ $\mathrm{m}=$ =mixer \\ $\mathrm{o}=$ initial, after expansion
}

\section{BOOK REVIEW}

The Chemist's English, by Robert Schoenfeld (Weinheim: VCH Verlagsgeselischaft, 1985) pp xii + 173. Price: DM42, US \$17.95 (ISBN 352726309 8).

This is a book for chemists, and other scientists, who have a genuine concern for the effective use of English in expressing their ideas in writing. It will be of little value to those without a good general knowledge of science, as many of the topics are discussed with particular reference to chemical concepts. For instance, the penultimate chapter is entitled 'A chemical analysis of the English sentence', and examines an English sentence as if it was a molecule and its words the constituent atoms. This is a novel idea and one that will probably be helpful to people accustomed to thinking along scientific lines.

The book consists of a series of 35 short essays on a wide variety of topics. Although not intended as a didactic book on English usage, the author's aim being to stimulate the reader into solving his problems for himself, it will prove a useful tool to the scientist wishing to improve his writ- ing. Mr Schoenfeld is himself a lucid writer, using crisp short sentences. He does not spare his reader detailed treatment of such complicated matters as gerunds, detached participles and word division in typesetting.

In examining many aspects of written English, the author shows how errors and solecisms can creep into even the most careful writing. There are a few usages that the author, like most editors, seems at first sight unaccountably hot against. Nevertheless he always presents a logical argument for his case, so the reader can formulate a defence if he stubbornly insists on maintaining a contrary view.

It is to be hoped that the book will also reach those scientists and technologists, seemingly in the majority, who regard the English language as a blunt instrument with which to batter their findings into the poor bloodied skulls of the reader. It is not unusual for the editor of this Journal to encounter sentences such as the following lquoted verbatim from a recently submitted paper): 'Figure 2 have been made up by writing the neperean logarithms of $K$ versus the opposites of the absolute temperature.' Mr Schoenfeld should be encouraged to write a companion volume starting at absolutely the first principles!

Unfortunately the author also feels the need to weave into his otherwise admirable prose a superfluous and overbearing mateyness of tone, a story-swapping waggishness more suited to the saloon bar. Thus, for example, he is not bashful of parading before us rather more about his own familiarity with China and Chinese, his knowledge of various ancient and modern European languages, his parentage and his sexual proclivities than we wish or need to know. Most of the essays were first published individually in various chemical journals, so this fault would not originally have been so apparent. But, when' reading large chunks of text at a time, the author's repeated elbow pokes leave the reader's sensibilities uncomfort ably bruised.

Overall, then, this is a book to be welcomed, but also to be taken in small portions.

PAUL DINSDALE 